\title{
UNSTEADY BOUNDARY LAYERS: CONVECTIVE HEAT TRANSFER OVER A VERTICAL FLAT PLATE
}

\author{
ROBERT A. VAN GORDER ${ }^{1}$ and K. VAJRAVELU ${ }^{\otimes 1}$
}

(Received 15 November, 2008; revised 23 April, 2009)

\begin{abstract}
In this paper, we extend the results in the literature for boundary layer flow over a horizontal plate, by considering the buoyancy force term in the momentum equation. Using a similarity transformation, we transform the partial differential equations of the problem into coupled nonlinear ordinary differential equations. We first analyse several special cases dealing with the properties of the exact and approximate solutions. Then, for the general problem, we construct series solutions for arbitrary values of the physical parameters. Furthermore, we obtain numerical solutions for several sets of values of the parameters. The numerical results thus obtained are presented through graphs and tables and the effects of the physical parameters on the flow and heat transfer characteristics are discussed. The results obtained reveal many interesting behaviours that warrant further study of the equations related to non-Newtonian fluid phenomena, especially the shear-thinning phenomena. Shear thinning reduces the wall shear stress.
\end{abstract}

2000 Mathematics subject classification: primary 76R10; secondary 76D10.

Keywords and phrases: unsteady boundary layers, coupled nonlinear ordinary differential equations, similarity solution, analytical solution, numerical solution.

\section{Introduction}

In 1997, Todd [9] analysed a two-dimensional laminar incompressible unsteady boundary layer flow over a flat plate by using an interesting similarity variable (referred to as the Blasius-Rayleigh-Stokes variable). Very recently Fang [2] studied the thermal boundary layer for the flat plate problem. The physical situation discussed by Todd and Fang is one of the possible cases. Another situation will be the study of convective heat transfer at a vertical flat plate, which plays an important role when the temperature difference between the surface and the ambient fluid is appreciably large. Hence it is interesting to study the effects of the density temperature variation on this new class of boundary layer flow.

\footnotetext{
${ }^{1}$ Department of Mathematics, University of Central Florida, Orlando, FL 32816, USA; e-mail: vajravel@ pegasus.cc.ucf.edu.

(C) Australian Mathematical Society 2009, Serial-fee code 1446-1811/2009 \$16.00
} 
We consider the two-dimensional laminar incompressible unsteady boundary layer flow over a vertical flat plate with leading edge accretion or ablation. We take the free stream velocity to be $U_{\infty}$, while the free stream temperature will be $T_{\infty}$. The $x$-axis is taken vertically up in the direction of the free stream, while the $y$-axis is perpendicular to the free stream. As discussed in [2,9], the equation of conservation of mass and the boundary layer equations governing the energy and momentum are

$$
\begin{gathered}
\frac{\partial u}{\partial x}+\frac{\partial v}{\partial y}=0, \quad \frac{\partial T}{\partial t}+u \frac{\partial T}{\partial x}+v \frac{\partial T}{\partial y}=\sigma \frac{\partial^{2} T}{\partial y^{2}}, \\
\frac{\partial u}{\partial t}+u \frac{\partial u}{\partial x}+v \frac{\partial u}{\partial y}=v \frac{\partial^{2} u}{\partial y^{2}}-g \beta\left(T-T_{\infty}\right),
\end{gathered}
$$

where $u$ and $v$ are the velocity components, in the $x$ and $y$ directions, respectively. Further, $T$ is the fluid temperature, $v$ is the kinematic viscosity, $\sigma$ is the thermal diffusivity, $g$ is the acceleration due to gravity and $\beta$ is the volumetric coefficient. The added second term in the momentum is called the buoyancy force term (in which we use the Boussinesq approximation $\left.\rho-\rho_{\infty}=-\rho \beta\left(T-T_{\infty}\right)\right)$. The addition of such a term to the momentum equation was considered in [10]. The appropriate conditions for (1.1) are

$$
u=0, v=0, T=T_{w} \text { at } y=0 ; \quad u \rightarrow U_{\infty}, T \rightarrow T_{\infty} \text { as } y \rightarrow \infty .
$$

As in [2], we use the stream function

$$
\psi(x, y, t) \equiv U_{\infty} \sqrt{(\cos \alpha) v t+(\sin \alpha)\left(v x / U_{\infty}\right)} f(\eta),
$$

with the similarity variable (referred to as the Blasius-Rayleigh-Stokes variable in [9])

$$
\eta \equiv \frac{y}{\sqrt{(\cos \alpha) \nu t+(\sin \alpha)\left(\nu x / U_{\infty}\right)}} .
$$

We also define a nondimensional temperature $\theta=\theta(\eta)$ as

$$
\theta(\eta) \equiv \frac{T-T_{\infty}}{T_{w}-T_{\infty}}
$$

Using (1.3)-(1.5), in System (1.1) and in Conditions (1.2), we get the coupled nonlinear system

$$
\begin{gathered}
f^{\prime \prime \prime}+\frac{1}{2}((\cos \alpha) \eta+(\sin \alpha) f) f^{\prime \prime}+G \theta=0, \\
\theta^{\prime \prime}+\frac{\operatorname{Pr}}{2}((\cos \alpha) \eta+(\sin \alpha) f) \theta^{\prime}=0, \\
f(0)=0, \quad f^{\prime}(0)=0, \quad \theta(0)=1, \quad f^{\prime}(\infty)=1, \quad \theta(\infty)=0,
\end{gathered}
$$

where

$$
G=\sqrt{(\cos \alpha) v t+(\sin \alpha)\left(v x / U_{\infty}\right)} g \beta\left(T_{w}-T_{\infty}\right) / U_{\infty}^{2}
$$

is the Grashof number or the free convection parameter and prime denotes differentiation with respect to $\eta$. We note that the similarity equation (1.6) is not a complete similarity equation, as the parameter $G$ depends on both variables $t$ and $x$. 
We now have three model parameters of interest: Pr the Prandtl number, $\alpha$ the leading edge accretion or ablation parameter, and $G$ the free convection parameter. The velocity components can be expressed in terms of the similarity variable $\eta$ as

$$
u=U_{\infty} f^{\prime}(\eta) \quad \text { and } \quad v=\frac{(v / 2)\left(\eta f^{\prime}(\eta)-f(\eta)\right) \sin \alpha}{\sqrt{(\cos \alpha) v t+(\sin \alpha)\left(v x / U_{\infty}\right)}} .
$$

We remark that unsteady boundary layers for other flow configurations have been discussed in a number of works, in addition to those references mentioned above (see, for instance, [1, 3-8, 11, 12]).

\section{Preliminaries}

In the absence of free convection currents (that is, when $G=0$ ), the system of equations (1.6)-(1.8) has solutions when $-\pi / 4 \leq \alpha<3 \pi / 4$, similar to the findings in [2,9]. In the case of $0<\alpha<\pi / 2$, we have leading edge accretion at the rate $U_{\infty} \cot \alpha$. For the case of $\pi / 2 \leq \alpha<\alpha^{*}$ (where $\alpha^{*} \approx 104.655^{\circ}$, as discussed in [2]), we have leading edge ablation at the rate $\left|U_{\infty} \cot \alpha\right|$. Finally, for the case $-\pi / 4 \leq$ $\alpha<0$, we have a downward boundary layer with trailing edge ablation. For various values of $G$, we obtain the flow and heat transfer characteristics for the above cases. The free convection parameter $G$ may be positive, zero, or negative. Physically, this means that the difference between the temperatures of the plate and the ambient fluid is positive, zero or negative, respectively.

2.1. The case of $\alpha=0$ In the special case of $\alpha=0,(1.6)$ and (1.7) reduce to the linear system

$$
f^{\prime \prime \prime}+\frac{\eta}{2} f^{\prime \prime}+G \theta=0, \quad \theta^{\prime \prime}+\frac{\operatorname{Pr}}{2} \eta \theta^{\prime}=0 .
$$

We can now obtain a solution for $\theta$ from (2.1). We find, upon integrating twice, that

$$
\theta(\eta)=1+\theta^{\prime}(0) \sqrt{\frac{\pi}{\operatorname{Pr}}} \operatorname{erf}\left(\frac{\sqrt{\operatorname{Pr}}}{2} \eta\right) .
$$

Using the expression for $\theta$ given in (2.2), we may then obtain $f$ from (2.1), namely,

$$
\begin{aligned}
f(\eta)= & f^{\prime \prime}(0) \int_{0}^{\eta} \int_{0}^{\xi} e^{-\zeta^{2} / 4} d \zeta d \xi \\
& -G \int_{0}^{\eta} \int_{0}^{\xi} e^{-\zeta^{2} / 4} \int_{0}^{\zeta}\left(1+\theta^{\prime}(0) \sqrt{\frac{\pi}{\operatorname{Pr}}} \operatorname{erf}\left(\frac{\sqrt{\operatorname{Pr}}}{2} \eta\right)\right) e^{\iota^{2} / 4} d \iota d \zeta d \xi .
\end{aligned}
$$

2.2. The case of $\alpha=\pi / 2$ In the special case of $\alpha=\pi / 2$, (1.6) and (1.7) reduce to

$$
f^{\prime \prime \prime}+\frac{1}{2} f f^{\prime \prime}+G \theta=0, \quad \theta^{\prime \prime}+\frac{\operatorname{Pr}}{2} f \theta^{\prime}=0 .
$$

While the ordinary differential equations are still coupled, note that repeated differentiation of (2.4) allows us to find $\theta^{\prime}$ and $\theta^{\prime \prime}$ in terms of $f$ and its derivatives. 
Substitution of these back into (2.4) gives

$$
f^{(v)}+\left(\frac{1+\operatorname{Pr}}{2}\right) f f^{(i v)}+f^{\prime} f^{\prime \prime \prime}+\frac{1}{2}\left(f^{\prime \prime}\right)^{2}+\frac{\operatorname{Pr}}{4} f^{2} f^{\prime \prime \prime}+\frac{\operatorname{Pr}}{4} f f^{\prime} f^{\prime \prime}=0 .
$$

From (2.5), we may obtain a series solution approximation to $f$ for small $\eta$, involving the unknown quantities $f^{\prime \prime}(0)$ and $\theta^{\prime}(0)$. Up to eighth order, we find that $f$ is given by

$$
\begin{aligned}
f(\eta)= & \frac{1}{2} f^{\prime \prime}(0) \eta^{2}-\frac{1}{6} G \eta^{3}+\frac{1}{24} G \theta^{\prime}(0) \eta^{4}-\frac{1}{240}\left(f^{\prime \prime}(0)\right)^{2} \eta^{5}+\frac{1}{360} G f^{\prime \prime}(0) \eta^{6} \\
& -\left(\frac{G^{2} f^{\prime \prime}(0) \theta^{\prime}(0)}{1440}+\frac{G^{2}}{2520}+\frac{G \operatorname{Pr} f^{\prime \prime}(0) \theta^{\prime}(0)}{10080}\right) \eta^{7} \\
& +\left(\frac{G^{2} \operatorname{Pr} \theta^{\prime}(0)}{80640}+\frac{G^{2} \theta^{\prime}(0)}{5376}+\frac{11\left(f^{\prime \prime}(0)\right)^{3}}{161280}\right) \eta^{8}
\end{aligned}
$$

where we have used the fact that (2.4) implies that $f^{(i v)}(0)=-G \theta^{\prime}(0)$. Employing (2.4) and (2.6), we obtain a series approximation to $\theta$, for small $\eta$ : up to fifth order, $\theta$ is given as

$$
\theta(\eta)=1+\theta^{\prime}(0) \eta-\frac{\operatorname{Pr}}{48} f^{\prime \prime} \theta^{\prime}(0) \eta^{4}+\frac{\operatorname{Pr} G}{240} \theta^{\prime}(0) \eta^{5}
$$

Note that, while we cannot specify the second derivative of $f$ at zero, $f^{\prime \prime}(0)$, nor the first derivative of $\theta$ at zero, $\theta^{\prime}(0)$, we can actually make statements about the values of the next highest derivatives for each function. Evaluating (2.4) at $\eta=0$, we see that

$$
0=f^{\prime \prime \prime}(0)+\frac{1}{2} f(0) f^{\prime \prime}(0)+G \theta(0)=f^{\prime \prime \prime}(0)+G
$$

and hence $f^{\prime \prime \prime}(0)=-G$. Similarly, $\theta^{\prime \prime}(0)=0$ gives

$$
0=\theta^{\prime \prime}(0)+\frac{\operatorname{Pr}}{2} f(0) \theta^{\prime}(0)=\theta^{\prime \prime}(0)
$$

2.3. Bounds on higher order derivatives when $\boldsymbol{G}=\mathbf{0}$ As was shown in [2], in the case where $G=0$, we may integrate (1.6)-(1.8) to find

$$
\begin{gathered}
f^{\prime}(\eta)=f^{\prime \prime}(0) \int_{0}^{\eta} \exp \left(-\frac{\cos \alpha}{4} t^{2}\right) \exp \left(-\frac{\sin \alpha}{4} \int_{0}^{t} f(\epsilon) d \epsilon\right) d t \\
\theta(\eta)=1-\frac{\int_{0}^{\eta} \exp \left(-(\operatorname{Pr} \cos \alpha) / 4 t^{2}\right) \exp \left(-(\operatorname{Pr} \sin \alpha) / 4 \int_{0}^{t} f(\epsilon) d \epsilon\right) d t}{\int_{0}^{\infty} \exp \left(-(\operatorname{Pr} \cos \alpha) / 4 t^{2}\right) \exp \left(-(\operatorname{Pr} \sin \alpha) / 4 \int_{0}^{t} f(\epsilon) d \epsilon\right) d t}
\end{gathered}
$$


By the evaluation of (2.8) as $\eta \rightarrow \infty$, we obtain

$$
\begin{gathered}
f^{\prime \prime}(0)=\left[\int_{0}^{\eta} \exp \left(-\frac{\cos \alpha}{4} t^{2}\right) \exp \left(-\frac{\sin \alpha}{4} \int_{0}^{t} f(\epsilon) d \epsilon\right) d t\right]^{-1}, \\
\theta^{\prime}(0)=-\left[\int_{0}^{\infty} \exp \left(-\frac{\operatorname{Pr} \cos \alpha}{4} t^{2}\right) \exp \left(-\frac{\operatorname{Pr} \sin \alpha}{4} \int_{0}^{t} f(\epsilon) d \epsilon\right) d t\right]^{-1} .
\end{gathered}
$$

In the literature, $f^{\prime \prime}(0)$ is known as the shear stress or skin friction at the wall, while $\theta^{\prime}$ is known as the temperature gradient or Nusselt number at the wall. Since $0 \leq f^{\prime}(\eta) \leq 1$ and $f(0)=0$, we have that $0 \leq f(\eta) \leq \eta$. We may use this bound on $f(\eta)$ to obtain bounds for $f^{\prime \prime}(0)$ and $\theta^{\prime}(0)$ via (2.9). We note that both lower and upper bounds depend on the sign of $\sin \alpha$. We have multiple cases, corresponding to the three possible intervals for $\alpha$, as discussed above. As $f^{\prime \prime}(0)$ and $\theta^{\prime}(0)$ are bounded, we suspect that analytical and numerical solutions may be obtained. We will then denote our choice of initial conditions as $\kappa=\kappa(\operatorname{Pr}, G, \alpha)=f^{\prime \prime}(0)$ and $\gamma=\gamma(\operatorname{Pr}, G, \alpha)=-\theta^{\prime}(0)$, where $\kappa$ and $\gamma$ satisfy the bounds discussed above. We now proceed to obtain analytical and numerical solutions for general parametric values of $\operatorname{Pr}, G$ and $\alpha$.

\section{Analytical solutions via Taylor series}

Let us assume that our solutions take the form

$$
f(\eta)=\sum_{j=0}^{\infty} A_{j} \eta^{j} \quad \text { and } \quad \theta(\eta)=\sum_{j=0}^{\infty} B_{j} \eta^{j}
$$

In practice, we can find no closed form relation for the coefficients in each series, due to the inherent nonlinearity of the system (1.6)-(1.7). As such, we will obtain a truncated series approximation to the solutions proposed above. For sufficiently small $\eta$, we consider fifth-order Taylor series in order to obtain approximate solutions to our system of ordinary differential equations; there are found to be:

$$
\begin{aligned}
f_{5}(\eta)= & \frac{\kappa}{2} \eta^{2}-\frac{G}{6} \eta^{3}+\frac{2 G \gamma-\cos \alpha}{48} \eta^{4}-\frac{\kappa^{2} \sin \alpha-2 \cos \alpha}{240} \eta^{5} \\
\theta_{5}(\eta)= & 1-\gamma \eta+\frac{\operatorname{Pr} \gamma \cos \alpha}{12} \eta^{3}+\frac{\operatorname{Pr} \kappa \gamma \sin \alpha}{48} \eta^{4} \\
& -\frac{\operatorname{Pr} G \gamma \sin \alpha+2 \operatorname{Pr}^{2} \gamma \cos ^{2} \alpha}{240} \eta^{5} .
\end{aligned}
$$

Note that for $\alpha=\pi / 2$, these series solutions agree with those obtained in (2.6) and (2.7), up to fifth order. Further, when $\alpha=0$, these solutions serve as approximations to the exact solutions provided in (2.2) and (2.3).

For small $\eta$, from the approximations in (3.1) we see that

$$
\frac{\partial f_{5}(\eta)}{\partial G} \approx \frac{1}{2} \frac{\partial \kappa}{\partial G} \eta^{2}-\frac{1}{6} \eta^{3} \text { and } \frac{\partial \theta_{5}(\eta)}{\partial G} \approx-\frac{\partial \gamma}{\partial G} \eta+\frac{\operatorname{Pr} \cos \alpha}{12} \frac{\partial \gamma}{\partial G} \eta^{3} \text {. }
$$



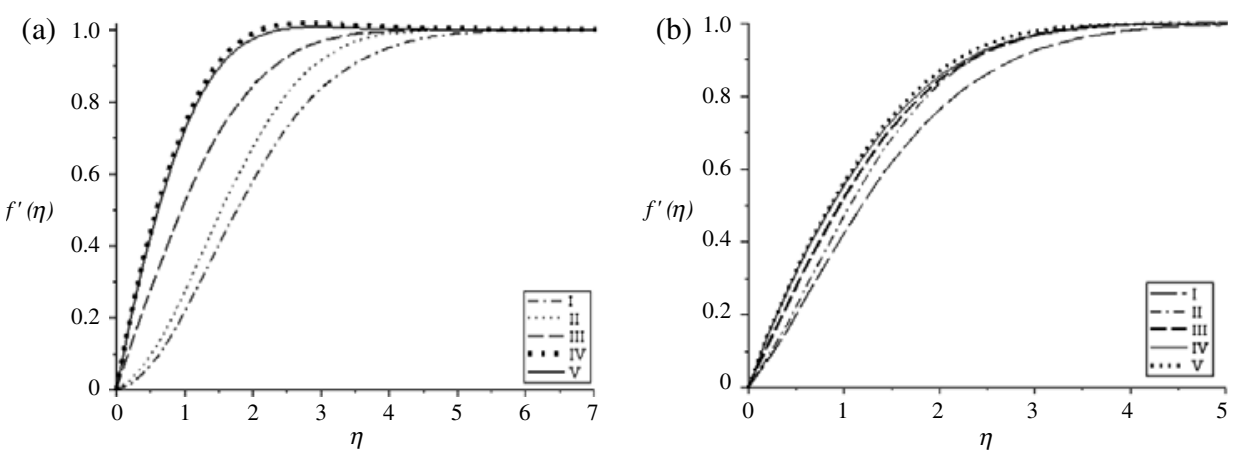

Figure 1. Profiles for $f^{\prime}(\eta)$ when (a) $\operatorname{Pr}=0.7$ and (b) $\operatorname{Pr}=7$; curves as in Table 1.

Thus, the influence of the free convection parameter $G$ on the approximate solutions (3.1) is more drastic on $\theta$ than on $f$, for small $\eta$. In the following section, we obtain numerical solutions to further study the influence of $G$ and $\alpha$ on the solutions to (1.6)-(1.8).

\section{Numerical solutions and discussion of results}

We use the boundary value problem solver (under dsolve in Maple 11) to obtain numerical solutions to the system of nonlinear coupled ordinary differential equations (1.6)-(1.8), to within a relative error tolerance of $10^{-5}$. We consider cases in which $\operatorname{Pr}=0.7$ (corresponding to air) and $\operatorname{Pr}=7$ (corresponding to water), for various values of the other parameters $\mathrm{G}$ and $\alpha$ (see Table 1 for different sets of values of the parameters considered).

In Figure 1(a), we plot the numerical solutions for $f^{\prime}(\eta)$ for $\operatorname{Pr}=0.7$ and in Figure 1(b) we do the same for $\operatorname{Pr}=7$. We provide similar plots for $f(\eta)$ and $\theta(\eta)$ in Figures 2 and 3, respectively. Note that values for all parameters considered in Figures 1-3 are given in Table 1. Also, in Table 2, we provide the values of $f^{\prime \prime}(0)$, for several sets of values of the parameters $G, \operatorname{Pr}$ and $\alpha$. In Table 3, similar results are presented for the Nusselt number, $\theta^{\prime}(0)$.

From Figure 3, we notice that the numerical solutions for $\theta(\eta)$ decrease as $G$ increases, for all $\eta$ considered (namely, $0 \leq \eta \leq 10$, where we set the numerical 'infinity' to 10). From Table 3, we see that

$$
\operatorname{sgn}\left(\frac{\partial \gamma}{\partial G}\right)=\operatorname{sgn}(\alpha)
$$

for those parametric values considered, where $\operatorname{sgn}(x)$ is -1 when $x<0$, 0 when $x=0$ and 1 when $x>0$. Then, for small values of Pr, we expect to have that

$$
\operatorname{sgn}\left(\frac{\partial \theta}{\partial G}\right)=-\operatorname{sgn}\left(\frac{\partial \gamma}{\partial G}\right)=-\operatorname{sgn}(\alpha) .
$$



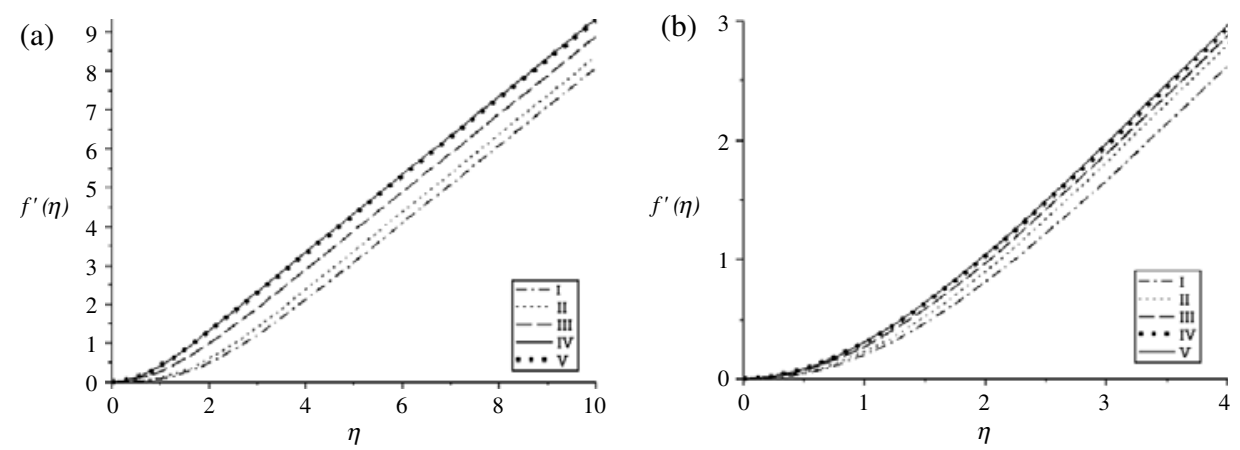

FIGURE 2. Profiles for $f(\eta)$ when (a) $\operatorname{Pr}=0.7$ and (b) $\operatorname{Pr}=7$; curves as in Table 1.
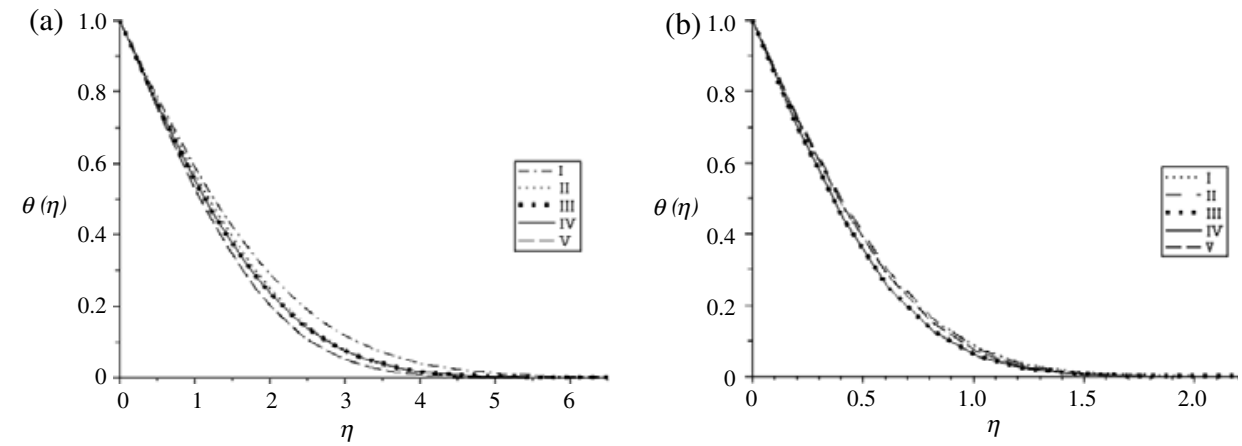

Figure 3. Temperature profiles $\theta(\eta)$ when (a) $\operatorname{Pr}=0.7$ and (b) $\operatorname{Pr}=7$; curves as in Table 1.

TABLE 1. Parameters and curve numbers.

\begin{tabular}{cccccc}
\hline & \multicolumn{5}{c}{ Curve number } \\
\cline { 2 - 6 } Parameter & I & II & III & IV & V \\
\hline$G$ & -0.5 & -0.5 & 0 & 0.5 & 0.5 \\
$\alpha$ & $-\pi / 8$ & $\pi / 4$ & 0 & 0 & $\pi / 4$ \\
\hline
\end{tabular}

That is, (for small $\operatorname{Pr}$ ) as we increase $G$, the change in $\theta(\eta)$ goes as the negative of the sign of $\alpha$. If $\alpha$ is negative, then $\theta(\eta)$ increases with an increase in $G$. However, if $\alpha$ is positive, then decreases when $G$ increases. When $\alpha$ is zero, there is no change in $\theta(\eta)$ when $G$ increases; this is shown in Table 3 where the value of $\theta^{\prime}(0)$ is fixed when $\alpha$ is zero. This behaviour is valid for sufficiently small values of Pr. Also, from Table 3 , we notice that $\theta^{\prime}(0)$ is always negative. Physically this means that the heat flow is only from the plate to the fluid. 
TABLE 2. Skin friction or values of $f^{\prime \prime}(0)$.

\begin{tabular}{cccccccc}
\hline & \multicolumn{3}{c}{$\operatorname{Pr}=0.7$} & & \multicolumn{3}{c}{$\operatorname{Pr}=7$} \\
\cline { 2 - 4 } \cline { 6 - 7 }$G / \alpha$ & $-\pi / 8$ & 0 & $\pi / 4$ & & $-\pi / 8$ & 0 & $\pi / 4$ \\
\hline-0.5 & 0.01725 & 0.12058 & 0.07436 & & 0.30653 & 0.38331 & 0.35117 \\
0 & 0.49931 & 0.56419 & 0.55287 & & 0.49931 & 0.56419 & 0.55287 \\
0.5 & 1.04198 & 1.00780 & 0.98294 & & 0.69525 & 0.74507 & 0.74898 \\
\hline
\end{tabular}

TABLE 3. Nusselt number or values of $\theta^{\prime}(0)$.

\begin{tabular}{cccccccc}
\hline & \multicolumn{3}{c}{$\operatorname{Pr}=0.7$} & & \multicolumn{3}{c}{$\operatorname{Pr}=7$} \\
\cline { 2 - 4 } \cline { 6 - 8 }$G / \alpha$ & $-\pi / 8$ & 0 & $\pi / 4$ & & $-\pi / 8$ & 0 & $\pi / 4$ \\
\hline-0.5 & -0.43053 & -0.47203 & -0.44482 & & -1.39882 & -1.49271 & -1.33694 \\
0 & -0.41223 & -0.47203 & -0.47029 & & -1.38938 & -1.49271 & -1.35450 \\
0.5 & -0.38768 & -0.47203 & -0.49282 & & -1.37954 & -1.49271 & -1.37104 \\
\hline
\end{tabular}

For all values of $\alpha$ considered, the numerical solutions for $f(\eta)$ and $f^{\prime}(\eta)$ increase with an increase in $G$ at each point $\eta$ in the domain (again, in our numerical solutions we consider $0 \leq \eta \leq 10$ ). Such a result is in agreement with (3.2). From Table 2 we see that

$$
\frac{\partial \kappa}{\partial G}>0 \stackrel{(3.2)}{\Longrightarrow} \operatorname{sgn}\left(\frac{\partial f^{\prime}}{\partial G}\right)=\operatorname{sgn}\left(\frac{\partial f}{\partial G}\right)=\operatorname{sgn}\left(\frac{\partial \kappa}{\partial G}\right)>0,
$$

for small $\eta$. As such, we see that (for small $\eta$ ) both $f(\eta)$ and $f^{\prime}(\eta)$ increase with an increase in $G$, as $\kappa \equiv f^{\prime \prime}(0)$ always increases with $G$.

\section{Conclusions}

Our primary focus was to understand the influence of the free convection parameter $G$ on the solution to the coupled system of nonlinear differential equations arising from a two-dimensional convection flow and heat transfer over a vertical flat plate with leading edge accretion or ablation. We note that for $G=0$, our solutions, both analytical and numerical, agree well with those in (1.3). In all cases considered, the analytical solutions provided in Section 3 agree very well with the numerical solutions, over a finite interval of convergence.

We find that the numerical solutions for $\theta(\eta)$ decrease as $G$ increases, over the numerical domain of $0 \leq \eta \leq 10$. Further, if $\alpha$ is negative, then $\theta(\eta)$ increases with an increase in $G$. However, if $\alpha$ is positive, then $\theta(\eta)$ decreases when $G$ increases. When $\alpha$ is zero, there is no change in $\theta(\eta)$ when $G$ increases. This behaviour is valid for 
sufficiently small values of Pr. Moreover, we find that $\theta^{\prime}(0)$ is negative for all values of the physical parameters. Physically, this means that the heat flow is only from the plate to the fluid.

For all values of $\alpha$ considered, the numerical solutions for $f(\eta)$ and $f^{\prime}(\eta)$ increase uniformly over the numerical domain of $0 \leq \eta \leq 10$ as the parameter $G$ increases. In general, the skin friction $f^{\prime \prime}(0)$ is an increasing function of the parameters $G, \operatorname{Pr}$ and $\alpha$. This information is useful in applications requiring the reduction of shear stress.

\section{Acknowledgement}

We thank the referees for their constructive comments which led to definite improvement in the paper.

\section{References}

[1] G. Amati, K. Koal, F. Massaioli, K. R. Sreenivasan and R. Verzicco, “Turbulent thermal convection at high Rayleigh numbers for a Boussinesq fluid of constant Prandtl number", Phys. Fluids 17 (2005) 121701.

[2] T. Fang, "A note on the unsteady boundary layers over a flat plate", Internat. J. Non-Linear Mech. 43 (2008) 1007-1011.

[3] S. Liao, "An analytic solution of unsteady boundary-layer flows caused by an impulsively stretching plate", Commun. Nonlinear Sci. Numer. Simul. 11 (2006) 326-339.

[4] S. Liao, "Series solutions of unsteady boundary-layer flows over a stretching flat plate", Stud. Appl. Math. 117 (2006) 239-263.

[5] A. Mehmood, A. Ali and T. Shah, "Heat transfer analysis of unsteady boundary layer flow by homotopy analysis method", Commun. Nonlinear Sci. Numer. Simul. 13 (2008) 902-912.

[6] R. Nazar, N. Amin and I. Pop, "Unsteady mixed convection boundary layer flow near the stagnation point on a vertical surface in a porous medium", Int. J. Heat Mass Transfer 47 (2004) 2681-2688.

[7] R. Nazar, N. Amin and I. Pop, "Unsteady boundary layer flow due to a stretching surface in a rotating fluid”, Mech. Res. Comm. 31 (2004) 121-128.

[8] I. Pop and T. Y. Na, "Unsteady flow past a stretching sheet", Mech. Res. Comm. 23 (1996) 413-422.

[9] L. Todd, "A family of laminar boundary layers along a semi-infinite flat plate", Fluid Dynam. Res. 19 (1997) 235-249.

[10] K. Vajravelu, "Convective heat transfer at a stretching sheet with suction or blowing", J. Math. Anal. Appl. 188 (1994) 1002-1011.

[11] J. C. Williams, J. C. Mulligan and T. B. Rhyne, "Semisimilar solutions for unsteady freeconvective boundary-layer flow on a vertical flat plate”, J. Fluid Mech. 175 (1987) 309-332.

[12] H. Xu, S. J. Liao and I. Pop, "Series solutions of unsteady three-dimensional MHD flow and heat transfer in the boundary layer over an impulsively stretching plate", Eur. J. Mech. B Fluids 26 (2007) 15-27. 